\title{
Gauge cooling for the singular-drift problem in the complex Langevin method - an application to finite density QCD
}

\author{
Keitaro Nagata ${ }^{* a}{ }^{\dagger}$ Hideo Matsufuru $^{a}$, Jun Nishimura ${ }^{a, b}$, Shinji Shimasaki ${ }^{c}$, \\ ${ }^{a}$ KEK, High Energy Accelerator Research Organization, 1-1 Oho, Tsukuba, Ibaraki 305-0801, \\ Japan \\ ${ }^{b}$ Graduate University for Advanced Studies (SOKENDAI), 1-1 Oho, Tsukuba, Ibaraki 305-0801, \\ Japan \\ ${ }^{c}$ Research and Education Center for Natural Sciences, Keio University, Hiyoshi 4-1-1, \\ Yokohama, Kanagawa 223-8521, Japan \\ E-mail: \\ matufuru@post.kek.jp, knagata@post.kek.jp, jnishi@post.kek.jp, \\ shinji.shimasaki@keio.jp
}

\begin{abstract}
We study full QCD at finite density and low temperature with light quark mass using the complex Langevin method. Since the singular drift problem turns out to be mild on a $4^{3} \times 8$ lattice we use, the gauge cooling is performed only to control the unitarity norm in this exploratory study. We report on our preliminary data obtained from the complex Langevin simulation up to certain Langevin time. While the data are still noisy due to lack of statistics, the onset of the baryon number density seems to occur at larger $\mu$ than half the pion mass, which is the value for the phase quenched QCD. The validity of our simulation is tested by the recently proposed criterion based on the probability distribution of the drift term.
\end{abstract}

34th Annual International Symposium on Lattice Field Theory

24-30 July 2016

University of Southampton, UK

${ }^{*}$ Speaker.

${ }^{\dagger}$ KEK-TH1941 


\section{Introduction}

In recent years we have witnessed remarkable progress in the study of systems with complex actions by the complex Langevin method (CLM). The CLM is based on the complexification of dynamical variables, which requires some conditions for the equivalence to the original theory. Although the conditions had not been known for a long time, Ref. [1,2] provided an explicit condition based on an argument for justification of the CLM. In order to satisfy this condition in the case of lattice QCD, the so-called gauge cooling was developed [3]. Thus, the complex Langevin simulation for QCD was realized in the QGP phase [4, 5] and in the heavy dense limit [6] up to quite large quark chemical potential, which goes far beyond the applicable limit of conventional approaches such as reweighting or the Taylor expansion. The validity of the gauge cooling was also proved explicitly [7] based on the argument for justification of the CLM. See also other contributions to this volume for recent studies on the CLM $[8,9,10,11]$.

In this work, we apply the CLM to QCD in the confined phase with light quarks. In this case, it is anticipated that the appearance of zero eigenvalues of the fermion matrix makes the drift term singular and spoils the validity of the CLM. This problem was shown to occur in some models $[12,13,14]$, and possible solutions were discussed $[15,16,17]$. In this work, we perform simulations on a $4^{3} \times 8$ lattice, where the singular drift problem turns out to be mild. For this reason, we use the gauge cooling only to control the unitarity norm instead of using it also to cure the singular drift problem as proposed in Refs. $[16,17]$. The validity of our simulation is tested by using a criterion that the probability distribution of the drift term should fall off exponentially or faster [18], which is required for the convergence property of a power series used in a refined argument for justification. This condition is considered a necessary and sufficient condition since it is slightly stronger than the previous condition required for the validity of the integration by parts $[1,2]$. The newly proposed criterion is shown to be useful not only in simple one-variable models [18] but also in systems with infinite degrees of freedom [19, 20].

We explain the framework of this work in the next section, provide preliminary results in section 3, and draw a temporal conclusion in the final section.

\section{Framework}

We study QCD on a four-dimensional Euclidean lattice with the lattice spacing $a$, the spatial extent $N_{s}$ and the temporal extent $N_{t}$. Introducing quark chemical potential $\mu$ makes the fermion determinant complex and spoils the importance sampling. We use the CLM to solve this problem. When one applies the Langevin method to a theory with a complex action, the dynamical variables in the theory have to be complexified. In lattice QCD, in particular, the space on which the link variables $U_{n \mu}$ take values should be extended from $\mathrm{SU}(3)$ to $\mathrm{SL}(3, \mathbb{C})$. Accordingly, we need to extend the action to a function of the complexified link variables in a holomorphic manner. In this work, we use the plaquette action

$$
S_{\mathrm{G}}=-\frac{\beta}{6} \sum_{x} \sum_{\mu>v} \operatorname{tr}\left[U_{x, \mu v}+U_{x, \mu v}^{-1}\right]
$$

for the gauge field, where $U_{x \mu} \in \mathrm{SL}(3, \mathbb{C})$ is a link variable and $U_{x, \mu v}$ is the plaquette given by $U_{x, \mu v}=U_{x \mu} U_{x+\hat{\mu} v} U_{x+\hat{v} \mu}^{-1} U_{x v}^{-1}$. Fermions are implemented using the standard staggered fermion 
formalism with the fermion matrix

$$
M(U, \mu)_{x y}=m a \delta_{x, y}+\sum_{v=1}^{4} \frac{\eta_{v}(x)}{2}\left[e^{\mu a \delta_{v 4}} U_{x v} \delta_{x+\hat{v}, y}-e^{-\mu a \delta_{v 4}} U_{x-\hat{v} v}^{-1} \delta_{x-\hat{v}, y}\right],
$$

which represents four flavors of quarks with the mass $m$ and the chemical potential $\mu$. Due to the relation $\varepsilon_{x} M(\mu)_{x y} \varepsilon_{y}=M^{\dagger}\left(-\mu^{*}\right)_{y x}$ with the "staggered $\gamma_{5}$ operator" $\varepsilon_{x}$, the fermion determinant becomes complex for real and nonzero $\mu$. As interesting observables, we measure the baryon number density $\langle n\rangle=\frac{1}{N_{V} N_{c}} \frac{\partial}{\partial(\mu a)} \log Z$ and the chiral condensate $\Sigma=\frac{1}{N_{V}} \frac{\partial}{\partial(m a)} \log Z$, where $N_{V}=$ $N_{s}^{3} N_{t}$. In measuring these observables in the CLM, they should also be extended to functions of complexified link variables in a holomorphic manner.

In the CLM including the gauge cooling procedure, the link variables are updated in the following two steps

$$
\begin{aligned}
\widetilde{U}_{x v}(t) & =g_{x} U_{x v}(t) g_{x+\hat{v}}^{-1} \\
U_{x v}(t+\varepsilon) & =\exp \left(i \sum_{a=1}^{N_{c}^{2}-1} \lambda_{a}\left[-v_{a x v}(\widetilde{U}) \varepsilon+\sqrt{2 \varepsilon} \eta_{a x v}\right]\right) \widetilde{U}_{x v}(t),
\end{aligned}
$$

where $t$ is the discretized Langevin time with the stepsize $\varepsilon$ and $\lambda_{a}$ are the Gell-Mann matrices. Eq. (2.4) represents the Langevin equation for the link variables. The Gaussian white noise $\eta_{\text {axv }}$ is real and normalized by $\left\langle\eta_{a^{\prime} x^{\prime} v^{\prime}}\left(t^{\prime}\right) \eta_{a x v}(t)\right\rangle_{\eta}=2 \delta_{a^{\prime} a} \delta_{x^{\prime} x} \delta_{v^{\prime} v} \delta_{t^{\prime} t}$, where the symbol $\langle\ldots\rangle_{\eta}$ represents an average over the noise with the Gaussian weight. The drift term is defined by

$$
v_{a x v}(U)=\lim _{\delta \rightarrow 0} \frac{S\left(e^{i \delta \lambda_{a}} U_{x v}\right)-S\left(U_{x v}\right)}{\delta},
$$

where $S=S_{G}-\frac{N_{f}}{4} \log \operatorname{det} M$ with $N_{f}=4$ in our case. At $\mu \neq 0$, the action $S$ is complex, and so is $v_{\text {axv }}$. Therefore, the use of Eq. (2.4) makes the link variables drift away from the SU(3) manifold in the non-compact directions.

In calculating the fermionic part of the drift term, we use the bilinear noise method, where the trace of $M$ is evaluated using the Gaussian noise vectors. It is known that the use of this method in the Langevin simulation does not give rise to systematic errors in the zero stepsize limit [21], which is in contrast to the situation in the hybrid $\mathrm{R}$ algorithm. While a naive implementation of this method violates the reality of the drift term at $\mu=0$, there is an improved implementation that makes the drift term real at $\mu=0$ [22], which we follow in our study.

Due to the complexification of dynamical variables, the gauge invariance of the action and the observables is enhanced from $\mathrm{SU}(3)$ to $\mathrm{SL}(3, \mathbb{C})$. The gauge cooling represented by Eq. (2.3) is performed by using a transformation for this enhanced symmetry. We determine $g_{x}$ in Eq. (2.3) in such a way that it reduces the unitarity norm $[4,5]$

$$
\mathscr{N}_{\mathrm{u}} \equiv \frac{1}{4 N_{V}} \sum_{x, v} \operatorname{tr}\left[\left(U_{x v}\right)^{\dagger} U_{x v}+\left(U_{x v}^{-1}\right)^{\dagger} U_{x v}^{-1}-2 \times \mathbb{1}_{3 \times 3}\right],
$$

which describes the deviation of $U_{x v}$ from SU(3) matrices. Note that the Hermitian conjugate in $\mathscr{N}_{\mathrm{u}}$ is taken after the complexification and that the unitarity norm is not invariant under an $\operatorname{SL}(3, \mathbb{C})$ transformation. 
In order to test the validity of our simulation, we calculate the probability of the drift term

$$
p(u)=\frac{1}{4 N_{V}}\left\langle\sum_{x v} \delta\left(u-u_{x v}\right)\right\rangle
$$

where $u_{x v}$ is the norm of the drift term for a link variable $U_{x v}$ defined by

$$
u_{x v}=\sqrt{\frac{1}{N_{c}^{2}-1} \sum_{a=1}^{N_{c}^{2}-1}\left|v_{a x v}\right|^{2}} .
$$

Since the drift term $v_{a x v}$ is calculated anyway in the CLM, the calculation of $p(u)$ does not require significant additional cost. In order for the CLM to be valid, the probability $p(u)$ should fall off exponentially or faster at large $u$ [18]. Note that the drift term $v_{a x v}$ contains the gauge field part and the fermionic part. A slow fall-off of each part represents the excursion problem and the singular drift problem, respectively. Thus the two problems that were thought to invalidate the CLM can be probed by the above criterion in a unified manner. Without this criterion, it is not obvious to tell whether either of these problems is really occurring or not.

\section{Results}

The parameters of the theory is chosen as $N_{s}^{3} \times N_{t}=4^{3} \times 8, m a=0.05$ and $\beta=5.7$, which corresponds to the confined phase at $\mu=0$. The chemical potential $\mu$ is varied in the range $\mu a \in$ $[0,0.65]$, which corresponds to $\mu / T \in[0,5.2]$. The Langevin simulations were performed with a fixed stepsize $\varepsilon=10^{-4}$ for the total Langevin time $t=30$. For the sake of comparison, we also study the phase quenched QCD, which is defined by replacing $\operatorname{det} M$ with $|\operatorname{det} M|$, using the (real) Langevin simulation with a stepsize $\varepsilon=2 \times 10^{-4}$ for the total Langevin time $t=20$. The Langevin time history of the unitarity norm is plotted in Fig. 1, which shows that the unitarity norm is under control during the simulations.

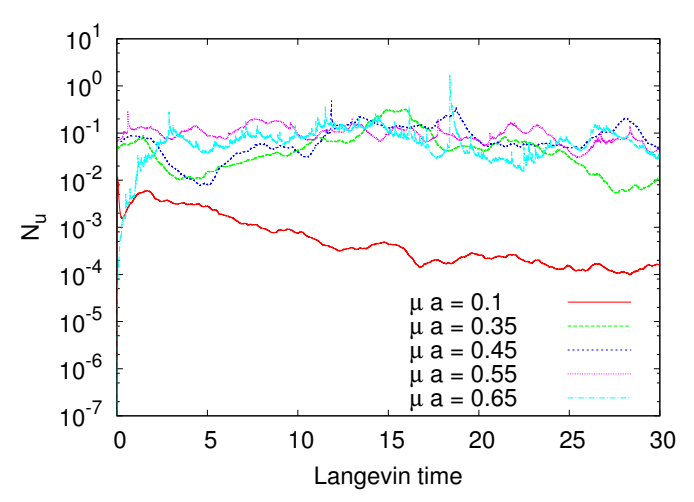

Figure 1: The Langevin time history of the unitarity norm for $0.1 \leq \mu a \leq 0.65$.

Figure 2 shows the $\mu$ dependence of the baryon number density and the chiral condensate obtained from the complex Langevin simulation of QCD. At small $\mu$, the baryon number density is zero and the chiral condensate is nonzero. As we increase $\mu$, the baryon number density starts to 

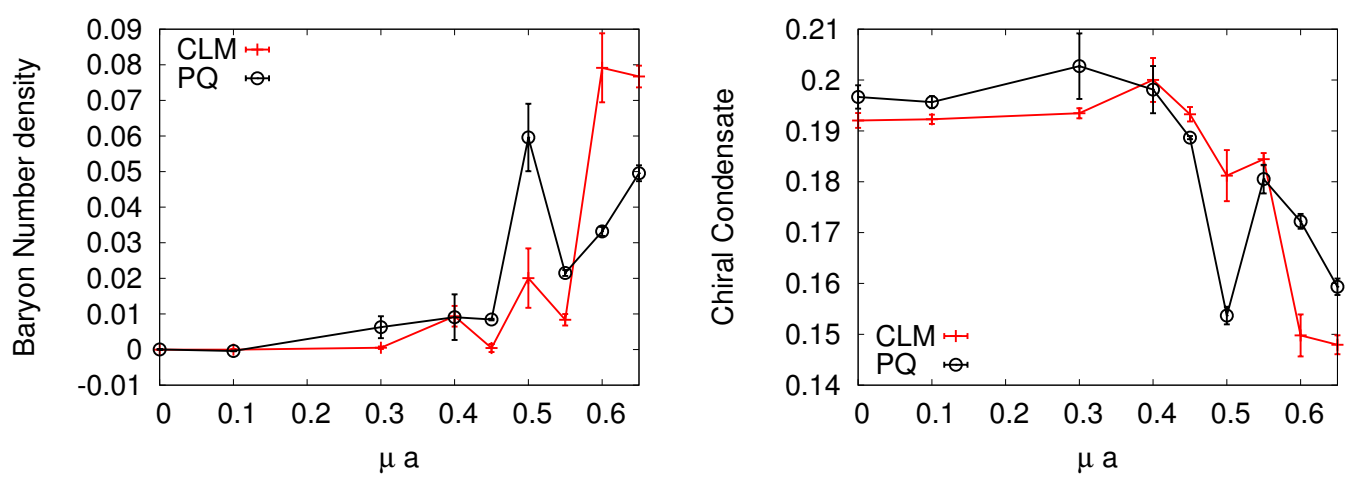

Figure 2: The baryon number density (Left) and the chiral condensate (Right) are plotted against $\mu a$. The symbol referred to as "CLM" represents the result of QCD with the CLM, while the symbol referred to as "PQ" represents the result of the phase quenched QCD with the real Langevin method.

increase and the chiral condensate starts to decrease at some point. At $\mu a=0.4 \sim 0.5$, the baryon number density reaches the order of 0.01 , which roughly corresponds to the density for having one baryon on the lattice, i.e., $1 / N_{s}^{3}=1 / 4^{3} \sim 0.016$.

In Fig. 2 we also plot the results of the real Langevin simulation for the phase quenched QCD (PQ). We observe clear tendency that the onset value of $\mu$ at which the baryon number density starts to increase is smaller for PQ. It is known that in PQ, the onset value is $\mu=m_{\pi} / 2$ [23], which can be estimated as 0.265 by using the results [24] of the mean field analysis for $m a=0.05$. In the case of QCD, on the other hand, the mean field analysis [24] yields an estimate of the onset value $\mu a=0.55$. Our results are in reasonable agreement with these predictions considering finite size effects, which smoothen the transition.
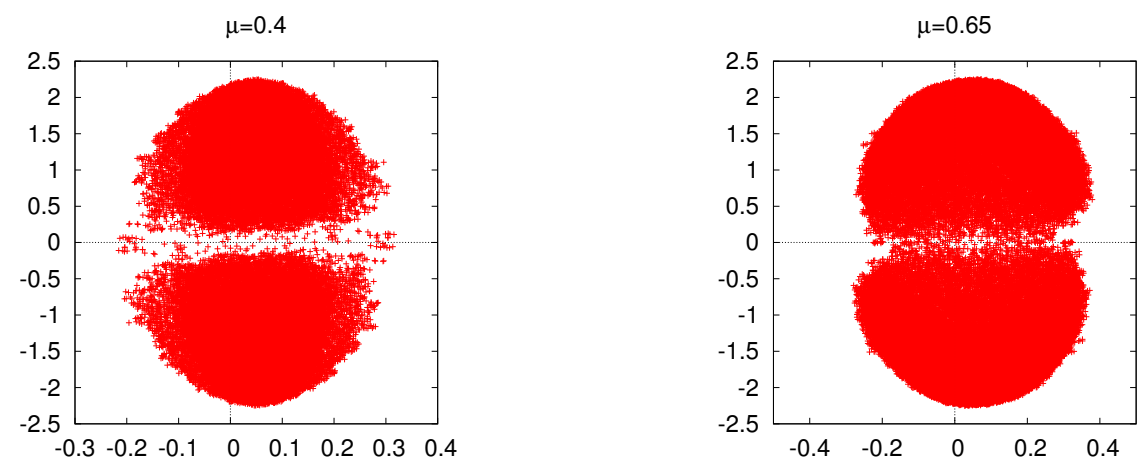

Figure 3: Scatter plots of the eigenvalues of the fermion matrix $M$ for $\mu a=0.4$ (Left) and 0.65 (Right).

The eigenvalue distribution of the fermion matrix $M$ is shown in Fig. 3 for $\mu a=0.4$ and $\mu a=0.65$. Let us recall that the eigenvalues close to the origin make the fermionic drift term large and cause the singular drift problem, which invalidates the CLM. In fact, we observe a gap along the real axis at $\mu=0$ due to finite size effects, although the lattice setup used in this work corresponds to the confined phase. As we increase $\mu$, the eigenvalue distribution extends in the 
real direction and the gap becomes smaller. At $\mu a=0.4$, the eigenvalue distribution still has a gap, which implies that there are not so many eigenvalues close to the origin. At $\mu a=0.65$, however, the gap is not clear any more, and there are quite a few eigenvalues close to the origin. At even larger $\mu$, the gap disappears eventually. Therefore, a crucial question that arises is: up to which value of $\mu$ our complex Langevin simulation is valid.
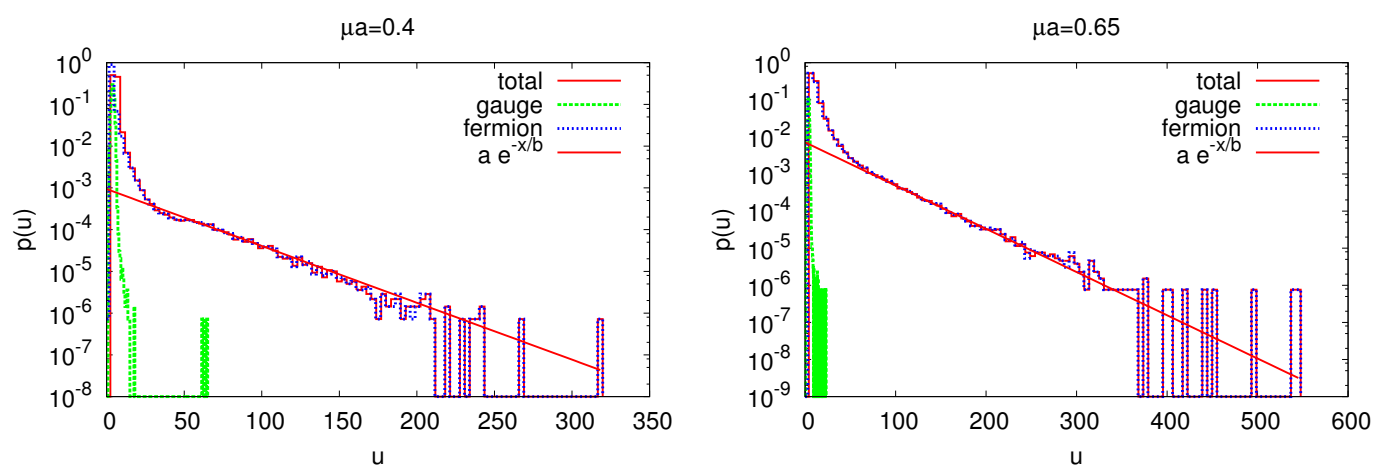

Figure 4: The probability distribution of the drift terms in the log scale. Straight lines represent fits to an exponential behavior $p(u)=a e^{-u / b}$.

In order to test the validity of our simulations, we use the criterion for correct convergence [18] based on the probability distribution $p(u)$ of the drift term, which we plot $p(u)$ in Fig. 4 for $\mu a=0.4$ and $\mu a=0.65$ in the log scale. We find that our results can be fitted by an exponential behavior $p(u)=a e^{-u / b}$, excluding the isolated peaks observed at large $u$, which are not statistically significant. We have performed similar fits for all values of $\mu$, and find that the probability distribution is suppressed exponentially at large $u$ for $\mu a \lesssim 0.65$. This implies that the condition for correct convergence [18] is satisfied in this region.

\section{Summary}

We performed complex Langevin simulations for lattice QCD at finite density in the confined phase on a $4^{3} \times 8$ lattice. The gauge cooling was used only for the unitarity norm in this exploratory study and it was found to stabilize the Langevin dynamics as in the case with the deconfined phase [4]. Comparing QCD and the phase quenched QCD, we find that the onset of the baryon number density occurs at larger $\mu$ in QCD than in the phase quenched QCD. The onset value for each case agrees reasonably with the predictions of the mean field analysis. We have also measured the probability distribution of the drift term, and confirmed that the criterion for correct convergence proposed recently is satisfied for $\mu a \lesssim 0.65$, which covers the interesting region in the present setup.

While our preliminary results are certainly encouraging, we also find that the auto-correlation time at $\mu a \gtrsim 0.4$ is quite long, which seems to be related the possible occurrence of the singular drift problem at $\mu a \gtrsim 0.7$. Furthermore, for a larger lattice, it is anticipated that the gap in the eigenvalue distribution of the fermion matrix disappears, which will cause a serious singular drift problem. We are currently trying to develop new techniques to overcome these problems. 


\section{Acknowledgments}

This work was supported by Grant-in-Aid for Scientific Research (No. 26800154 for K.N. and No. 23244057, $16 \mathrm{H} 03988$ for J.N.) from Japan Society for the Promotion of Science. Simulations were mainly carried out on SX-ACE at RCNP Osaka University and CRAY XC40 at YITP Kyoto University. SR16000 at KEK was also used for developing a parallel code. S.S. was supported by the MEXT-Supported Program for the Strategic Research Foundation at Private Universities "Topological Science" (Grant No. S1511006).

\section{References}

[1] G. Aarts, E. Seiler, and I.-O. Stamatescu, Phys. Rev. D81 (2010) 054508.

[2] G. Aarts, F. A. James, E. Seiler, and I.-O. Stamatescu, Eur. Phys. J. C71 (2011) 1756.

[3] E. Seiler, D. Sexty, and I.-O. Stamatescu, Phys.Lett. B723 (2013) 213-216.

[4] D. Sexty, Phys.Lett. B729 (2014) 108-111.

[5] Z. Fodor, S. D. Katz, D. Sexty, and C. Török, Phys.Rev. D92 (2015) no.9, 094516.

[6] G. Aarts, F. Attanasio, B. Jäger, and D. Sexty, arXiv:1610.04401.

[7] K. Nagata, J. Nishimura, and S. Shimasaki, PTEP 2016 (2016) no.1, 013B01, arXiv: 1508.02377.

[8] D. K. Sinclair and J. B. Kogut, arXiv:1611.02312.

[9] S. Schmalzbauer and J. Bloch, arXiv:1611.00702.

[10] G. Aarts, E. Seiler, D. Sexty, and I.-O. Stamatescu, arXiv:1611.02930.

[11] F. Attanasio and B. Jaeger, arXiv:1610.09298.

[12] A. Mollgaard and K. Splittorff, Phys.Rev. D88 (2013), no. 11116007.

[13] J. Greensite, Phys. Rev. D90 (2014), no. 11114507.

[14] J. Nishimura and S. Shimasaki, Phys. Rev. D92 (2015), no. 1011501.

[15] A. Mollgaard and K. Splittorff, Phys.Rev. D91 (2015), no. 3036007.

[16] K. Nagata, J. Nishimura, and S. Shimasaki, PoS LATTICE2015 (2016) 156, [arXiv:1511.08580].

[17] K. Nagata, J. Nishimura, and S. Shimasaki, JHEP 07 (2016) 073.

[18] K. Nagata, J. Nishimura, and S. Shimasaki, arXiv:1606.07627.

[19] K. Nagata, J. Nishimura, and S. Shimasaki, a contribution to this volume.

[20] Y. Ito and J. Nishimura, arXiv:1609.04501.

[21] G. G. Batrouni, G. R. Katz, A. S. Kronfeld, G. P. Lepage, B. Svetitsky and K. G. Wilson, Phys. Rev. D 32 (1985) 2736.

[22] D. K. Sinclair and J. B. Kogut, PoS LATTICE2015 (2016) 153.

[23] D. T. Son and M. A. Stephanov, Phys. Atom. Nucl. 64 (2001) 834-842.

[24] I. Barbour, N.-E. Behilil, E. Dagotto, F. Karsch, A. Moreo, M. Stone, and H. W. Wyld, Nucl. Phys. B275 (1986) 296-318. 